\title{
Optimal algorithms for the batch scheduling problem in OBS networks
}

\author{
Gustavo B. Figueiredo ${ }^{a}$, Eduardo Candido Xavier ${ }^{b}$, Nelson L. S. da Fonseca ${ }^{b}$ \\ ${ }^{a}$ Department of Computer Science, Federal University of Bahia, Brazil \\ ${ }^{b}$ Institute of Computing, University of Campinas, Brazil
}

\begin{abstract}
This paper introduces optimal batch scheduling algorithms for the problem of scheduling batches of bursts in optical burst switching networks. The problem is modelled as a job scheduling problem with identical machines. The consideration of previously scheduled bursts in the scheduling allows such modeling. Two optimal algorithms with polynomial time complexity are derived and evaluated. Results show that the algorithm that allows re-scheduling of previously scheduled bursts leads to preferred solutions.

Moreover, an extended version of the JET reservation protocol is proposed for efficient handling of batches of bursts. Results obtained via simulation prove the superior performance of the BATCHOPT algorithm.
\end{abstract}

Keywords: OBS Networks, channel scheduling, batch scheduling

\section{Introduction}

In Optical Burst Switching (OBS) networks, packets are aggregated at edge nodes to create transmission units called bursts. A control packet is transmitted out-of-band, ahead of the burst, so that bandwidth can be reserved for the data burst. The control packet carries information about the burst, such as size and offset time (defined as the time interval separating the arrival of the control packet and that of the data burst). A scheduling mechanism then reserves bandwidth of the output channels of nodes in the core network for incoming bursts, based on the information carried by their control packets. Since the nodes at the network border do not wait for the confirmation of bandwidth reservation to transmit a burst, the incoming burst will be discarded at a core node if bandwidth has not been reserved.

The Just-Enough-Time (JET ) [1] is a commonly used protocol for resource reservation in OBS networks. JET reserves the channel for the duration of the transmission of a burst, starting at its expected arrival time (given by the offset time minus the burst processing time). If this request for bandwidth reservation is granted, a new offset time is calculated, and this information is inserted into the control packet being forwarded to the next hop in the route.

Since bursts have different offset times, they may arrive in a different order than that of their control packets. This can lead to fragmentation of the occupancy of the output channels, since the occupancy pattern of output channels typically alternates between periods of occupancy and period of idleness, called void intervals. These void intervals can be used to accommodate the transmission of new bursts. Indeed, a void interval, $I_{j}$, defined by its starting, $s_{j}$, and its ending time, $e_{j}$, can be allocated to a burst with an arrival time, $t^{\prime}$, and departure time, $t^{\prime \prime}$ if and only if $s_{j} \leq t^{\prime} \mathrm{e}$ $t^{\prime \prime} \leq e_{j}$

Most of the existing algorithms for burst scheduling provide greedy processing to individual bursts $[41,42,43,48$, 28]. However, this approach can lead to the loss of bursts which could be avoided if the arrival time were previously known. One way of ameliorating this type of loss is to gather reservation requests during a certain time interval, and then schedule them as a batch of requests. The objective of this process is to maximize the number of bursts transmitted, i.e, to minimize the loss of bursts. For this reason, the occupancy of the output channels by the bursts can be modeled as an interval graph [56] with the solution of the burst assignment problem given by the solution of the coloring of an interval graph problem.

Email addresses: gustavo@dcc.ufba.br (Gustavo B. Figueiredo), ecx@ic.unicamp.br (Eduardo Candido Xavier), nfonseca@ic. unicamp.br ( Nelson L. S. da Fonseca) 
Previous work [11] [12] on batch scheduling in OBS networks have addressed the channel reservation problem as a job scheduling problem with non-identical machines. However, such modeling leads to an NP-hard problem. As a consequence, heuristics have been proposed to solve the problem. In this paper, we show how the problem of batch scheduling in OBS networks can be formulated as a job scheduling problem with identical machines. We, then, introduce two optimal algorithms: one for networks with no prioritized requests and which has linear time complexity and the other for networks with prioritized requests which involve polynomial time complexity. In addition to low computational complexity, the results derived via simulation show that the proposed algorithms produce a lower probability of blocking than existing heuristics. These algorithms differ in respect to the re-scheduling of previously scheduled bursts. Moreover, the JET protocol is extended to operate efficiently with batch scheduling algorithms. Such an extension is compatible with the JET protocol, since its operation is exactly that of the JET protocol for scheduling individual bursts.

This paper is organized as follows. Section 2 presents concepts related to batch scheduling. Section 3 presents related background and notation. Section 4 reviews related work. Section 5 presents the new optimum batch scheduling algorithms. Section 6 presents an extended version of the JET protocol. Section 7 presents numerical examples and Section 8 draws some conclusions.

\section{Batch Scheduling in OBS networks}

To minimize the chance of loss of burst, an algorithm should allocate bandwidth for a burst so that the chances of allocation to upcoming bursts is maximized. A channel is considered available for accommodation of a burst if there is a void large enough to accommodate the request. If no such channel is available, the request will be lost.

The scheduling algorithms proposed in $[41,42,43,48,28]$ employ greedy strategies to reserve resources, but these strategies use only the information on individual control packets when they arrive without consideration of the overall demand during intervals between control packet arrivals.
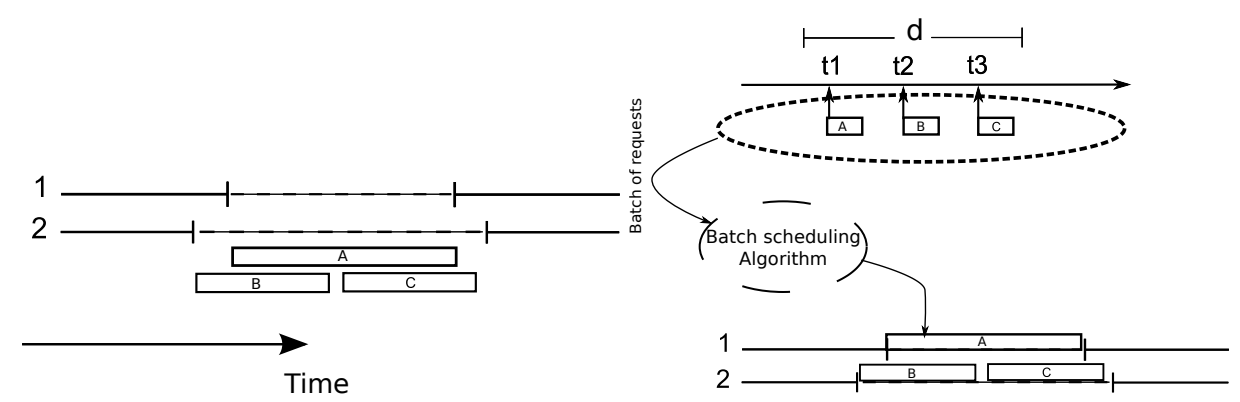

(a) Example of bad instances for greedy strategies.

(b) Batch scheduling of channels leading to no losses.

Figure 1: Examples of how batch scheduling can avoid losses of bursts

Figure 1(a) provides an example of a situation in which greedy algorithms fail to allocate resources for bursts: Let $\mathrm{A}, \mathrm{B}$ and $\mathrm{C}$ be control packets arriving in this order and let their corresponding bursts arrive in that same order. These bursts can be accommodated only in channels 1 and 2 (dashed lines). Note that if channel 2 were used for burst $\mathrm{A}$ and channel 1 for burst B (as could happen if the the algorithms proposed in [28, 43, 42] were used) there would be no way to accommodate burst C. On the other hand if channel 1 is used by burst A and channel 2 by bursts B and C, the result is a no loss scenario. This example illustrates the failure of scheduling due to lack of knowledge about future requests.

To decrease the loss of bursts, a new class of batch scheduling algorithms were proposed in [11] and [12] . The idea here is to group the largest possible number of control packets and process them together. After gathering information on the control packets, they are ordered and assigned to the channel with the smallest index value that has available wavelength to accommodate the burst (Figure 1(b)). One main characteristic of the heuristics presented in [11] and [12] is that they collect requests arriving in time intervals of a fixed duration before processing them. This strategy, however, generates losses when the beginning of batch processing succeeds a request starting time. 


\section{Background and Notation}

The batch scheduling problem in OBS networks (denoted BS-OBS) can be stated as follows: Let $M$ be a set of $k$ channels, and let $I$ be a batch of $n$ requests formed by control packets. On each channel $m_{i} \in M$ there can be previously scheduled requests and voids resulting from lack of channel occupancy. These can be used to allocate the requests in order to maximize the number of requests granted. Each request in the control packet $J_{x}=\left(s_{x}, e_{x}, w_{x}\right) \in I$ is identified by a 3-tuple; the arrival time of the burst, $\left(s_{x}\right)$, the finishing time of the burst, $\left(e_{x}\right)$ and the request weight indicating the priority of the scheduling burst, $\left(w_{x}\right)$. The objective is to find an allocation for a subset of requests such that the sum of the weights of the requests granted is maximized.

The offset time of a control packet $\left(s_{x}, e_{x}, w_{x}\right)$ arriving at time $t$ is given by $s_{x}-t$. The control packet must be processed during this offset time, otherwise its corresponding burst will be lost.

Next, some definitions will be introduced to facilitate the understanding of the mathematical formulation of the BS-OBS problem.

Let $G=(V, E)$ be a graph, where $V(G)$ represents the set of vertices of $G$ and $E(G)$ the set of edges. Given a vertex $u \in V(G)$, we define the adjacency/neighborhood of $u$ by $\operatorname{Adj}(u)=\{v \in V(G) ;(u, v) \in E(G)\}$. A subgraph $H$ of $G$ is a graph such that $V(H) \subseteq V(G)$ and $E(H) \subseteq E(G)$. The degree of vertex $u$ in the graph $G$, denoted by $d(u \mid G)$, is the size of the set $\operatorname{Adj}(u)$. The subgraph $H$ of $G$ is induced by $V(H)$ if for every pair $u, v \in V(H)$ we have that $(u, v) \in E(H)$ if and only if $(u, v) \in E(G)$. A clique of a graph $G$ is a set $C \subseteq V(G)$ such that $\forall u, v \in C ;(u, v) \in E(G)$. A clique $C$ is maximal if there is no other clique $C^{\prime}$ in $G$ such that $C \subset C^{\prime}$ [56].

The graph $G$ is called an interval graph if there is a correspondence/bijection between the set of vertices and a set of intervals on the real line, such that there is an edge between two vertices if and only if the correspondent intervals intersect, i.e, $(u, v) \in E(G) \Leftrightarrow I_{v} \cap I_{u} \neq \emptyset$ [56]. Interval graphs have several properties that can be used to solve problems in combinatorics. The fact that interval graphs can be recognizable and colorable in linear time will be explored in this paper.

Interval graphs are typically used in the solution of the job scheduling problem, which can be stated as follows: let $I=\left\{J_{1}=\left(s_{1}, e_{1}, w_{1}\right), \ldots, J_{n}=\left(s_{n}, e_{n}, w_{n}\right)\right\}$ be a list of $n$ jobs. Moreover, there are $k$ machines with the same processing capacity that are used to process these jobs. Initially, all machines are free starting at time 0 . The problem is to select a sub-list $I^{\prime} \subseteq I$ with maximum total weight such that no pair of jobs allocated to the same machine intersect their processing intervals.

This problem is known as the job scheduling problem with identical machines (denoted by S-IM) since there is no restriction on which machine each job can be processed; otherwise it is called job scheduling problem with non-identical machines (denoted by S-NIM). In S-NIM problem, there are restrictions on the assignment of jobs to machines, i.e, for each job $J_{i}$ there is a list $N_{i}$ of machines on which that job cannot be scheduled. The solution of the BS-OBS problem introduced here is based on an S-IM formulation in which jobs are represented by the requests for bandwidth allocation and machines are represented by the output channels of core nodes.

\section{Related Work}

In [11], four heuristics for the solution of the OBS scheduling problem were introduced. All the heuristics involved time complexity $O(n k \log (N))$, where $n$ is the number of requests being processed, $k$ the number of channels and $N$ the number of previously allocated requests. In these heuristics, requests are modeled as interval graphs $G$. They are briefly described below:

Smallest Vertex Ordering (SLV): In SLV, requests are allocated in the smallest last order, i.e, the first request allocated is the one corresponding to vertex $v_{1}$. The vertices $v_{1}, \ldots, v_{n}$ of a graph $G$ are considered to be ordered in a smallest last fashion if $v_{i}$ has the smallest degree in the subgraph induced by the vertices $v_{1}, \ldots, v_{i}$. If a request cannot be allocated in any channel, it is discarded and the process is repeated for all other vertices on that ordering.

The idea behind the SLV algorithm is that if a graph has only a few vertices with large degree, then if these requests are allocated first, a small number of channels is used for them. Nonetheless, SLV can generate great loss of requests as reported in [11]. The problem with this heuristic is illustrated in Figure 2. The requests are shown in Figure 2(a) and the corresponding interval graph is presented in Figure 2(b). Vertex "A" is the one with the highest degree in the smallest last ordering, and consequently the first one to be processed. However, scheduling "A" generates the loss of all the other requests. 


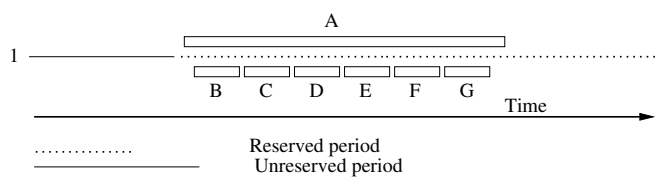

(a) Requests over the time

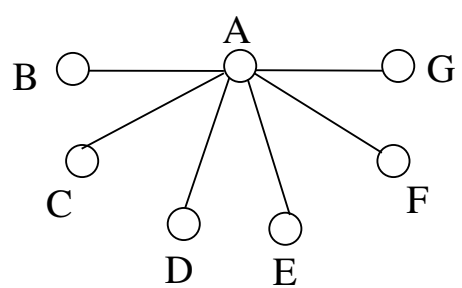

(b) Correspondent interval graph

Figure 2: Example where heuristic in [11] is inadequate.

Maximal Cliques First (MCF): The MCF heuristic computes the order in which the requests are going to be processed and also the requests that will be discarded. This heuristic computes all the maximal cliques of $G$ and then sorts them chronologically. Let $\left\{C_{1}, C_{2}, \ldots, C_{m},\right\}$ be the set of maximal cliques of $G$ ordered in a way such that $C_{i}<C_{j}$ for $i<j$. The algorithm processes the requests of cliques in increasing order. If the size of a clique $C_{j}$ exceeds the number of channels $k$, then the $\left|C_{j}\right|-k$ requests with smallest finishing time are discarded, since necessarily $M-k$ requests are discarded if there is a clique of size $M$ in the interval graph such that $k<M$.

This heuristic can also produce poor results. Considering the example presented in Figure 2, when the heuristic MCF is executed, the first clique to be processed (see Figure 2) is the clique with vertices "A" and "B". Since there is only one channel, request " $\mathrm{A}$ " is scheduled and all the remaining requests are discarded.

Smallest Start-time First Ordering (SSF): with this heuristic, requests are ordered and processed according to their starting time. Poor results can also be produced as illustrated by the example in Figure 2. In this example the first request to be processed is request " $\mathrm{A}$ " and all other requests are discarded.

Largest Interval First Ordering $(L I F)$ : with this heuristic, requests are ordered and processed in non-increasing order of duration. Considering again the example presented in Figure 2, it is possible to see the poor behavior of this heuristic. Once "A" is allocated, all other requests are discarded. It is interesting to note that even when the total length of the other requests is greater than the size of "A", all other requests are discarded.

These heuristics depend on the structure of the interval graphs generated; for some graphs good solutions can be found, but not for others. Thus, the main problem with all of the proposed approaches is that there is no guarantee that the best solution will be achieved.

In [12] Charcranoon et al. present a heuristic that schedules the maximum number of requests, considering only one channel. This heuristic Max Stable Set algorithm (Max-SS) [12] is presented next. It tries to find a maximum independent set to maximize the number of disjoint requests that can be scheduled on a channel. Max-SS finds one independent set for each channel, with a time complexity of $O(n \log n)$ which is the complexity needed to find the maximum independent set in an interval graph.

\section{Optimum algorithms for the BS-OBS problem}

The job scheduling formulation of the BS-OBS problem relies on the list $I$ of reservation requests as well as the list $S$ of requests already allocated to the channels. The problem is to find a subset of requests of $I$ with a maximum total weight that can be scheduled on the channels, since two intersecting requests cannot be scheduled on the same channel.

The heuristics in [11] were based on the formulation of the job scheduling problem with non-identical machines. Thus, if some incoming request intersects a request already scheduled to a machine $m$, then the second request cannot be scheduled for this machine. 
[13] introduces an optimal algorithm for the job scheduling problem with non-identical machines which has computational complexity of $O\left(n^{k+1}\right)$. However, its computational complexity is prohibitive for the solution of the BS-OBS problem because the exponential dependency on the number of channels $(k)$.

The approach proposed here is the formulation of the BS-OBS problem as a job scheduling with identical machines problem. For that, instead of trying to accommodate requests of $I$ into the existing voids generated by previously allocated requests $S$, all requests in the set $I \cup S$ are considered for scheduling. Moreover, all previous allocated requests in $S$ are rescheduled. In this way, the BS-OBS problem can be formulated as a S-IM problem.

Figure 3 illustrates this idea. At a given moment, channel 1 is reserved for the time period $[8,14]$ and channel 2 , for the time period [3,9] and [15,20] then a set of incoming requests A ([1,6]), B ([10,14]), C ([16, 21]) and D $([7,12])$ arrived. These incoming requests are grouped with those previously allocated in a single batch, and both channels become elegible for allocation by any of the requests of this newly formed batch.

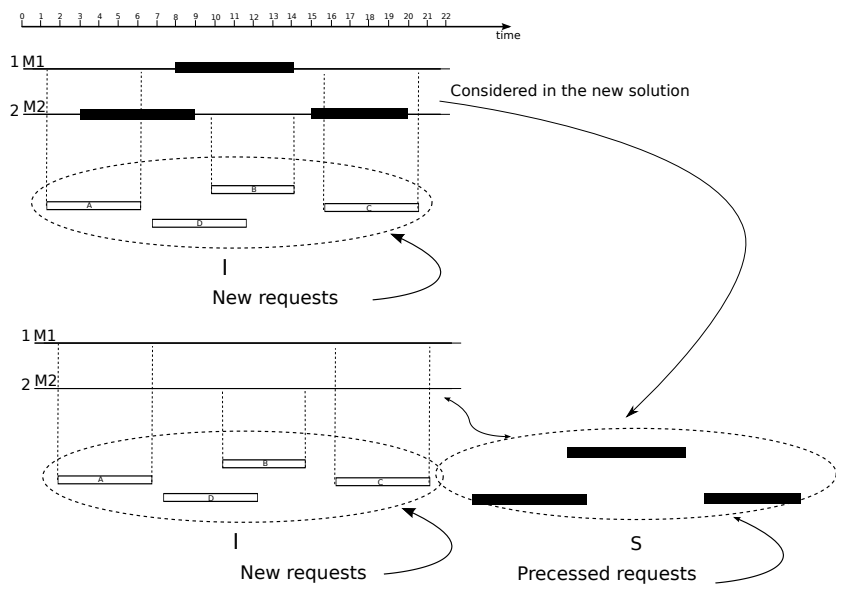

Figure 3: Batch Scheduling with Identical Machines.

\subsection{Optimum algorithm with linear time complexity}

A formulation for the batch scheduling problem with linear time complexity is proposed here. The algorithm, called GreedyOPT, is suitable for networks in which all requests have the same priority. It involves two major steps. In the first, the problem is transformed into a job scheduling problem with identical machines, thus allowing the employment of fast exact algorithms.

In the second step, the algorithm in [6], proposed for job scheduling with identical machines, with time complexity $O(n \max \{\log (n), k\})$ is used. This algorithm process sequentially the requests of the newly formed batch. It tries to schedule the requests on one of the channels. If a given request cannot be scheduled, the algorithm tries to replace it with one of the requests already scheduled that has latest ending time. The correctness of the algorithm is given by Theorem 1.

Theorem 1. If one of the requests in a set has its duration shortened by the anticipation of its ending time, the number of requests granted is equal to or higher than the number of requests in the original allocation for that set of requests.

Proof. See page 147 in [2].

GreedyOPT considers both the already allocated requests and the incoming new ones for the new schedule. GreedyOPT processes the new requests in chronological order to accommodate them into one of the $k$ channels. If this is not possible, the requests already allocated but with a data burst yet to arrive can be deallocated if a new assignment decision will improve the burst loss ratio. A heap is used to sort the requests, which leads to a $O(S \log (S))$ complexity for this step. The GreedyOPT is presented in Algorithm 1.

The GreedyOPT algorithm optimally solves the batch scheduling problem with identical machines when all requests have the same priority. This is shown in Theorem 2. 


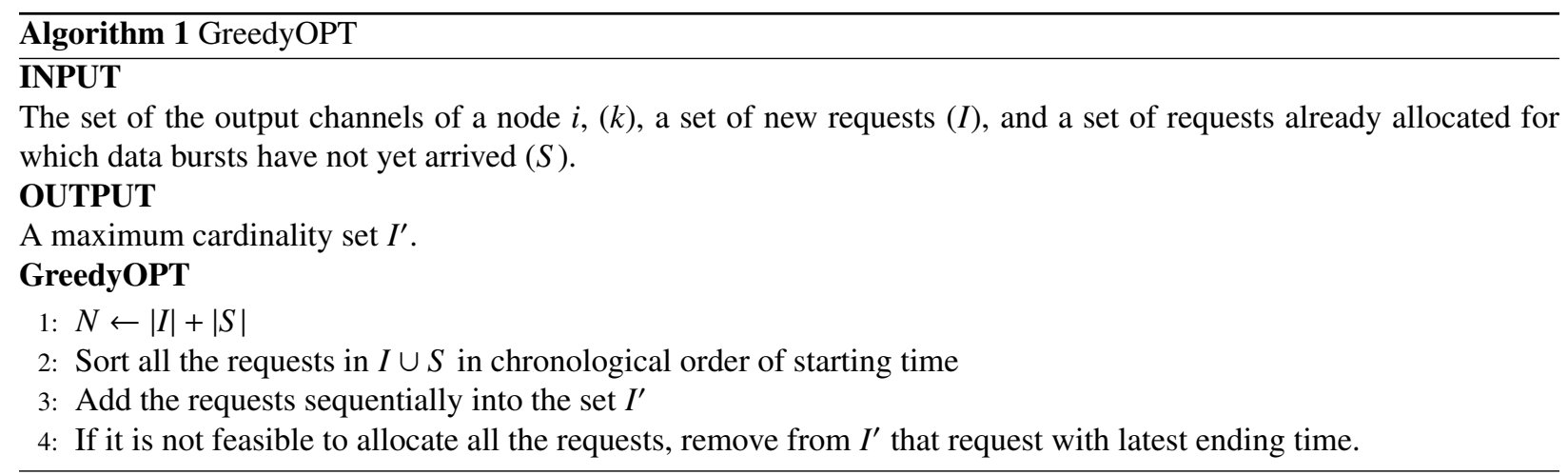

Theorem 2. Let $t_{0}$ be the earliest time at which more than $k$ requests in $J$ intersect each other and $e_{y}=\max _{j \in J} e_{j}$. Then, the optimal schedule excludes this yth request.

Proof. See Proposition 1 in [6]

The next theorem establishes the computational complexity of GreedyOPT.

Theorem 3. The computational complexity of the GreedyOPT algorithm is $O(N \log (N)+N k)=O(N \max (\log (N), k))$, where $k$ is the number of output channels of an OBS node, $n$ the number of incoming requests, $S$ the set of requests already allocated with the data burst yet to arrive $s=|S|$, the cardinality of the set $S$ and $N=n+s$.

Proof. See Appendix A.

\subsection{Optimal algorithm with polynomial time for prioritized requests}

\section{Eduardo INI}

This section, presents an algorithm with polynomial time complexity, entitled BATCHOPT, for networks in which requests have different priorities. As with the GreedyOPT algorithm, it is implemented in two steps. In the first step, the problem is transformed into a problem of job scheduling with identical machines. In the second step, an adaptation of the algorithm presented below is used to schedule the requests.

Arkin and Silverberg [13] proposed an optimal algorithm (denoted here $A S$ ) for the job scheduling problem with identical machines which will be described next. Our algorithm extends the $A S$ algorithm to include the set $S$ of requests already scheduled in the solution.

$A S$ builds an interval graph $G$ considering incoming requests and it computes all the maximal cliques of this graph; it then sorts the cliques into an increasing order for the starting time, $C_{1}, \ldots, C_{r}$. Figure 4 exemplifies this construction.
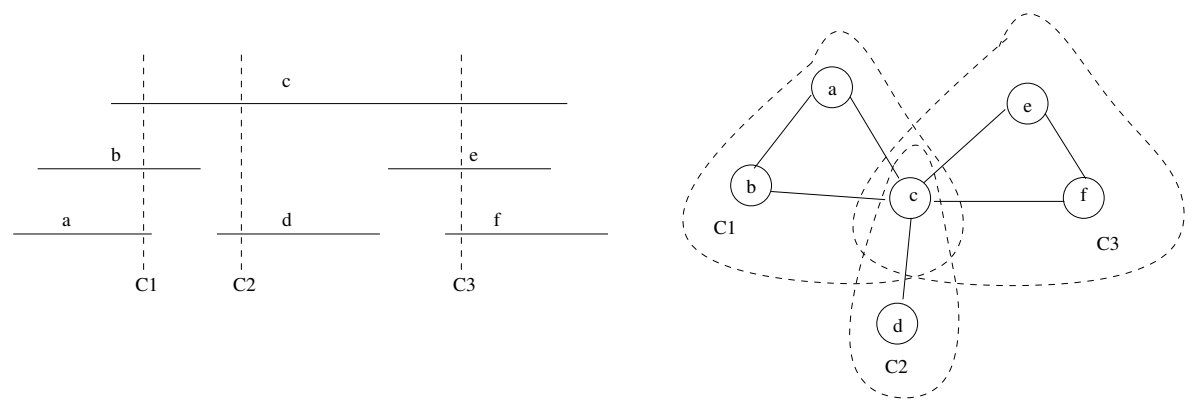

Figure 4: Requests are on the left. On the right is the corresponding interval graph and its maximal cliques numbered according to the ordering of time. 
A flow-graph $G^{\prime}$ is then constructed as follows: first create a vertex $v_{0}$ and for each clique $C_{j}(j=1, \ldots, r)$ create a vertex $v_{j}$, then, create directed $\operatorname{arcs}\left(v_{j}, v_{j-1}\right)$ for each $C_{j}$, with cost 0 and infinite capacity. Let $M$ be the maximum size of a clique among the cliques $C_{1}, \ldots, C_{r}$. For each clique $C_{j}$, create a directed arc $\left(v_{j-1}, v_{j}\right)$ with cost 0 and capacity equal to $M-\left|C_{j}\right|$ that represents a dummy job. For each job $J_{i}$ belonging to cliques $C_{j}, \ldots, C_{j+l}$, create a directed arc $\left(v_{j-1}, v_{j+l}\right)$ with capacity 1 and cost $w_{i}$. This arc represents all cliques which $J_{i}$ belongs $\left(C_{j}\right.$ to $\left.C_{j+l}\right)$. The aim is to find a flow from $v_{0}$ to $v_{r}$ in $G^{\prime}$ of $(M-k)$ units and with minimum cost. In the solution to this minimum cost flow problem in $G^{\prime}$, the arcs along the flow correspond to jobs that must be discarded whereas arcs with zero flow correspond to jobs that should be scheduled.

Figure 5 exemplifies the construction of the flow-graph $G^{\prime}$ for the interval graph $G$ in Figure 4 . It is created four vertexes, $v_{0}$ and three vertexes associated with cliques: $v_{1}$ corresponding to $C_{1}, v_{2}$ corresponding to $C_{2}$ and $v_{3}$ corresponding to $C_{3}$. Then it is created the bottom $\operatorname{arcs}\left(v_{3}, v_{2}\right),\left(v_{2}, v_{1}\right),\left(v_{1}, v_{0}\right)$ with cost 0 and infinite capacity. The maximum size of a clique in this example is $M=3$ (cliques $C_{1}$ and $C_{3}$ ). Notice that $M=\left|C_{1}\right|=\left|C_{3}\right|$. Then it is not created dummy arcs for these cliques, since the capacity of the arcs should be $M-\left|C_{1}\right|=M-\left|C_{3}\right|=0$. It is created only one dummy arc $\left(v_{1}, v_{2}\right)$ for $C_{2}$, with capacity 1 and cost 0 . Now it is created the arcs associated with jobs. Since job $a$ belongs only to $C_{1}$, it is created an arc $\left(v_{0}, v_{1}\right)$ with capacity 1 and cost $w_{a}$. Similarly it is created arcs associated to jobs $b, d, e$, and $f$, since each one of these jobs belongs to only one clique. The exception is job $c$, that belongs to $C_{1}, C_{2}$ and $C_{3}$, and in this case an arc $\left(v_{0}, v_{3}\right)$ is created, but also with capacity 1 and cost $w_{c}$. The construction of the flow graph is then finished. The problem is then to find a minimum cost flow from $v_{0}$ to $v_{3}$ of $3-k$ units of flow.

Eduardo FIM

Figure 5: An example of construction of a flow graph.

Theorem 4. The algorithm AS [13] optimally solves the job scheduling problem with identical machines.

Proof. See [13] for the proof.

To include already scheduled jobs in the $A S$ algorithm, it is necessary to have a formulation that can be applied to BS-OBS.

The set $S$ of previously scheduled requests can be easily introduced in the S-IM problem by assuming that they have a weight of infinity for each request $J_{i} \in S$. This guarantees that the already scheduled requests will remain scheduled in the final solution. The formulation of the proposed algorithm, entitled BATCHOPT, considers both the requests to be scheduled and those already scheduled in the search for an optimal solution, as in [13]. The pseudo-code of the BATCHOPT algorithm is presented next (Algorithm 2).

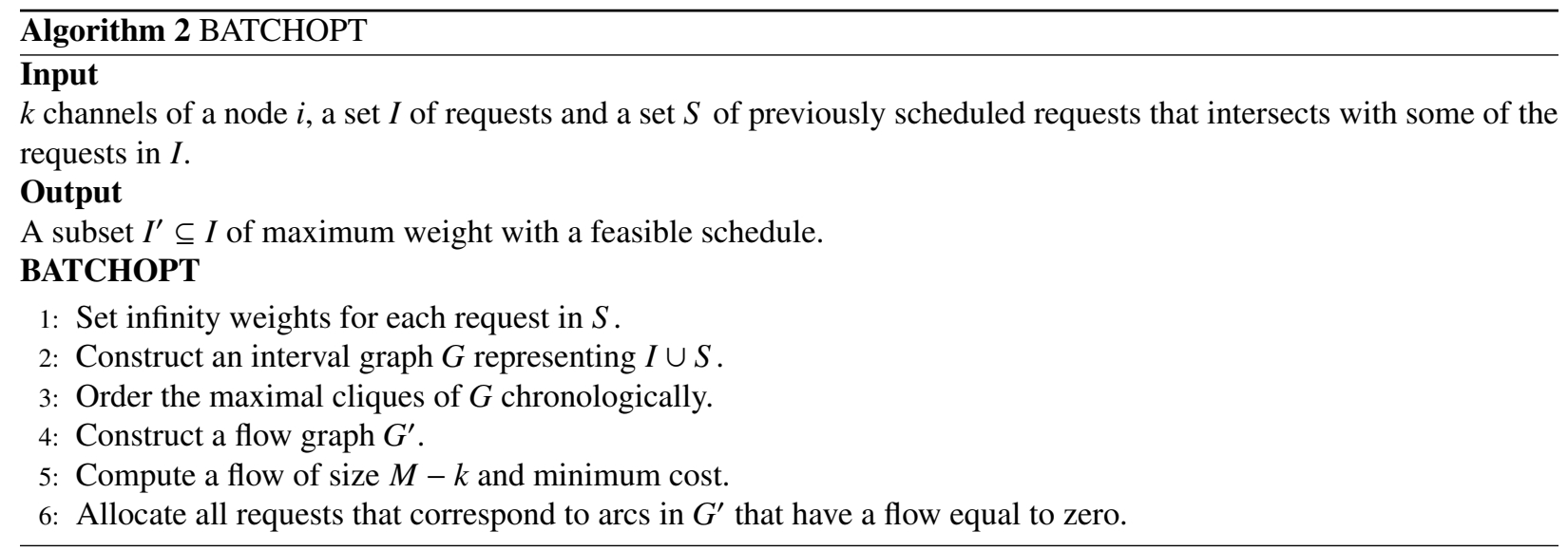

The optimality and feasibility of the schedule produced by the algorithm can be proved using the following theorem. 
Theorem 5. The BATCHOPT algorithm optimally solves the OBS scheduling problem. Moreover, each request already being scheduled remains scheduled on its previously scheduled channel.

Proof. See Appendix B

We now establish the time complexity of the proposed algorithm.

Theorem 6. The BATCHOPT algorithm has time complexity $O\left(N^{2} \log (N)+N\right)$, where $N=|I \cup S|$, i.e, $N$ is the number of requests in the batch plus the number of requests in $S$.

Proof. See Appendix B.

\section{Adaptation of the JET protocol}

In this section, a variation of the JET protocol adapted for batch scheduling is proposed.

The batch scheduling algorithms previously proposed collect requests during a time window, called acceptance window, and then employ various heuristics to schedule the requests. It was assumed that each intermediary node have a fixed acceptance window $\Delta$ used to gather requests to form a batch. However, such an approach leads to the unnecessary loss of bursts, since the fact that requests can arrive at a network node with different offset times is ignored. Figure 6 illustrates this type of loss. In this figure, two control packets arrive during the fixed size time window. Burst $A$ arrive at time $t_{A}$ and the burst $B$ arrive at time $t_{B}$. While burst $A$ will be processed by the batch scheduling policy and will be potentially transmitted, burst $B$ will not be considered by the scheduling policy and it will be lost, because it arrived during the fixed time window.

To overcome this problem, we propose an extension of the JET protocol, called JET- $\Delta$, which is employed jointly with batch scheduling algorithms. In this variation, the acceptance window is added to the offset time, as illustrated in Figure 7. Furthermore, the instant of time that ends the period for collecting requests and begins the processing period, called the processing threshold, is determined in a way that it occurs prior to the arrival of the next arriving burst, ensuring that all requests are processed before the arrival of their burst.

In the JET protocol, the source node computes the offset time based on the estimated processing time of all intermediary nodes along the route to the destination node. The offset time computed at the source node $s$ with destination $d$, along a route with intermediary nodes $H$ is

$$
T_{d}^{s}=\left(\sum_{i \in H} P_{i}\right)+P_{d}
$$

where $P_{i}$ is the estimated processing time at an intermediary node $i$ and $P_{d}$ is the processing time at the destination.

When an intermediary node $i \in H$ receives a control packet, it processes the request. If the request is scheduled then it computes a new offset time decreasing its processing time, and sends this information in the control packet to the next node in the route. Let $T_{d}^{i}$ be the offset time of the control packet received at time $t_{d}$ by node $i$ which has $d$ as a destination. Node $i$ computes, at time $t_{i}$, a new offset time as:

$$
T_{d}^{i+1}=T_{d}^{i}-\left(t_{i}-t_{d}\right)
$$

The processing time $\left(t_{i}-t_{d}\right)$, at node $i$ is then decreased.

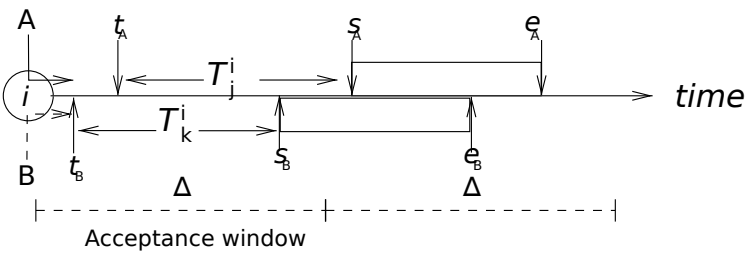

Figure 6: Example of data burst loss due to the use of fixed size acceptance window. 
A worst case estimation of the computation of the offset time at the source node is assumed, which leads to an offset time at the source node computed os:

$$
T_{d}^{s}=\left(\sum_{i \in I} P_{i}\right)+|I| \Delta+P_{d} .
$$

$T_{d}^{s}$ includes the value $|I| \Delta$, which accounts for all acceptance time windows of all intermediary nodes. This offset time should be enough to avoid losses, since no node can take more time than $\left(P_{i}+\Delta\right)$ to process one control packet. In general, the processing time of each node is of the order of microseconds, and the acceptance window is of the order of milliseconds. In this case, the processing times are almost negligible, and an upper bound $P_{\max }$ can be established.

Since at any given OBS node there can be several control packets from distinct source-destination pairs waiting to be processed and these control packets can have different offset times, it is important to ensure that each control packet will be processed before the arrival of its corresponding burst. Thus, a processing threshold $(L)$ should be determined by the data burst arriving first and this should be computed for every arrival of a control packet. The threshold is given by $L=\left(t_{R}+\Delta\right)-\delta$, where $R$ is the $\min _{r}\left\{t_{r}+T_{j}^{i}+\Delta\right\}, \delta$ is the batch processing time, and $t_{r}$ the arrival time of control packet $r$ with offset time $T_{j}^{i}$ at node $i$ on its way to node $j$. The request which determines the processing threshold is the request for the burst which arrives first.

It is important to note that the JET- $\Delta$ extension can be used by batch scheduling algorithms, as well as by greedy scheduling algorithms, by making $\Delta=0$.

When the processing threshold is reached, the batch is processed. All bursts going to the same destination are grouped and the information about the new batch goes into the same control packet to decrease the overhead [8].

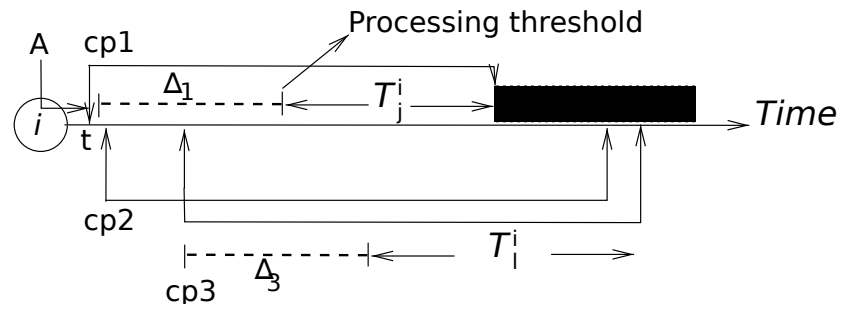

Figure 7: Time diagram of the JET- $\Delta$ reservation protocol

The inclusion of the acceptance window into the offset time can increase the end-to-end delay experienced by the packets assembled in a burst. However, this can be ameliorated if the maximum tolerable end-to-end delay is considered when computing the acceptance window as:

$$
\Delta=\frac{D-T_{j}^{s}}{H}
$$

Where $D$ is the maximum tolerable end-to-end delay, $T_{j}^{s}$ the offset time of a burst $j$ at the network border and $H$ is the number of hops from source to destination.

An alternative way of reducing the introduction of delay is to use prediction of burst size at the assembly edge node, as proposed in [22] and [21].

\section{Numerical Examples}

In this section, the proposed algorithms are compared to existing algorithms, although the algorithm MAX-SS is not considered since it considers only OBS networks with a single data channel. To evaluate the performance of the algorithms, simulations were carry out using the simulator OB2S (Optical Burst Switching Simulator). The algorithms were implemented in $\mathrm{C}$, using the library available in [4]. 
Each simulation run consisted of the allocation of 10.000 requests to the available channels. Each experiment was executed 20 times, with different seeds and the confidence interval for the mean value was computed using a confidence level of $95 \%$.

Two distinct scenarios were considered in the simulations: in the first scenario, the experiments reported in [11] were reproduced in order to evaluate the performance of the algorithms and compare the results those in the literature. In the second scenario, topologies of real networks with a more realistic set of parameters were employed. The results are reported in the following subsections.

\subsection{Topology with one bottleneck node}

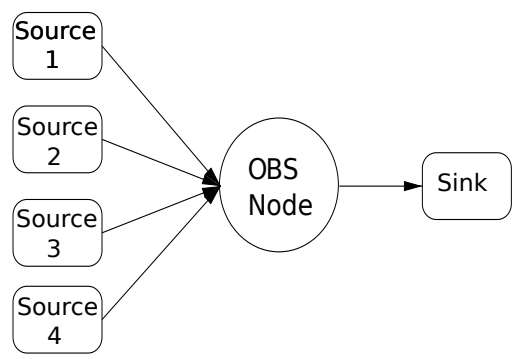

Figure 8: Topology used in the first simulation scenario.

Figure 8 shows the topology used in the first scenario. In this topology, there is a single OBS node connected to four sources and a single destination node. Each input link has 2 wavelengths (one for data and one for control signaling). The link connecting the OBS node to the destination has five wavelengths (four for data and one for control signaling). Each wavelength has 2.5 Gbps capacity (OC-48).

As in [11], we defined the constant $\tau$ as the time required to transmit 1024 bits on one of the wavelengths, i.e., $\tau=\frac{1024}{2377728000}=4.3 e-7$ seconds. Bursts were generated according to a Poisson distribution with mean size, $\bar{b}=81920$ bits. The offset time was generated according to an uniform distribution in the interval $[130 \tau, 150 \tau]$. The acceptance window had an arbitrary value of $100 \tau$, which was approximately $40 \mu \mathrm{sec}$.

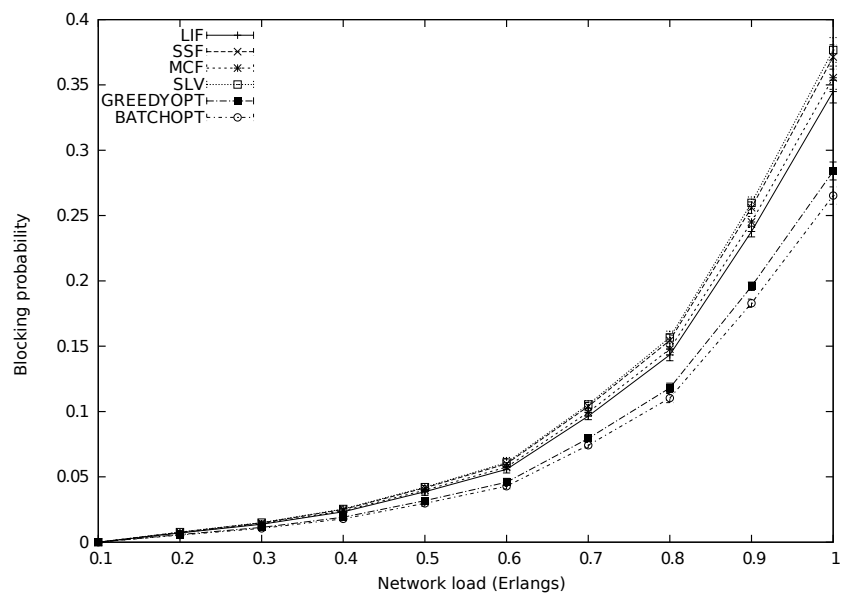

Figure 9: Blocking probability for each algorithm.

Figure 9 shows the blocking probability resulted from the algorithms. The lowest blocking probability is produced by the BATCHOPT algorithm, followed by the GreedyOPT algorithm. Although both BATCHOPT and GreedyOPT optimally schedule requests for a given batch, only the BATCHOPT guarantees that requests already scheduled in previous batches remain scheduled in the current computation. As a consequence, only the new requests which do not overlap with already scheduled requests are added to the final solution. With GreedyOPT algorithm, requests already 
scheduled in previous batches may be dropped during the computation of future batches, thus increasing the blocking probability.

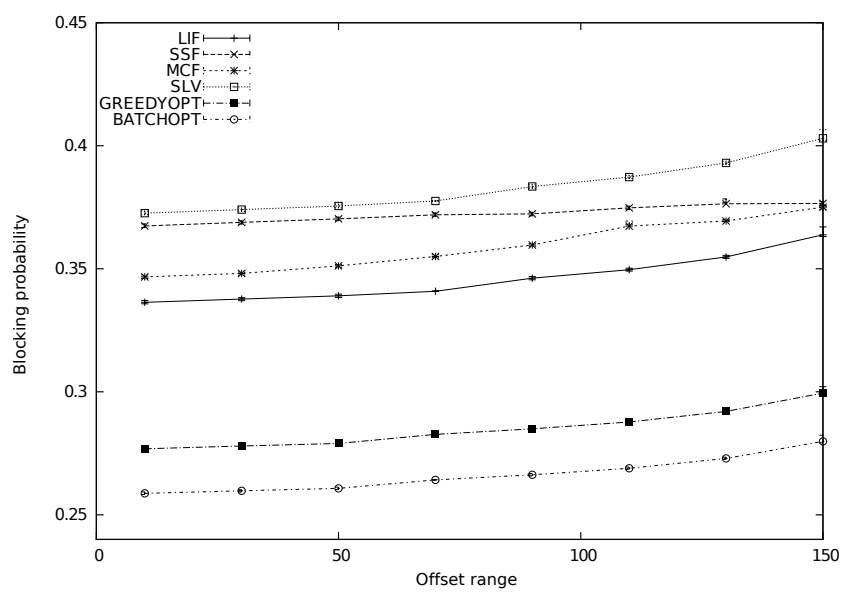

Figure 10: Blocking Probability as a function of the difference in offset time

Another experiment conducted using this simulation scenario aimed to evaluate the effect of the offset range in the probability of blocking. The offset range is defined as the difference between the highest and lowest value of the offset time, $T_{\max }-T_{\min }$. The value of $T_{\max }$ was fixed at $200 \tau$ and the value of $T_{\min }$ was gradually increased. In this experiment, the network load was of $99 \%$ of the link capacity.

Figure 10 shows that, as observed in the experiments reported in [11], there is an increase in blocking probability as the offset range is increased due to the retro-blocking phenomenon of the JET signaling protocol, in which a reservation request can be blocked by another reservation starting after it.

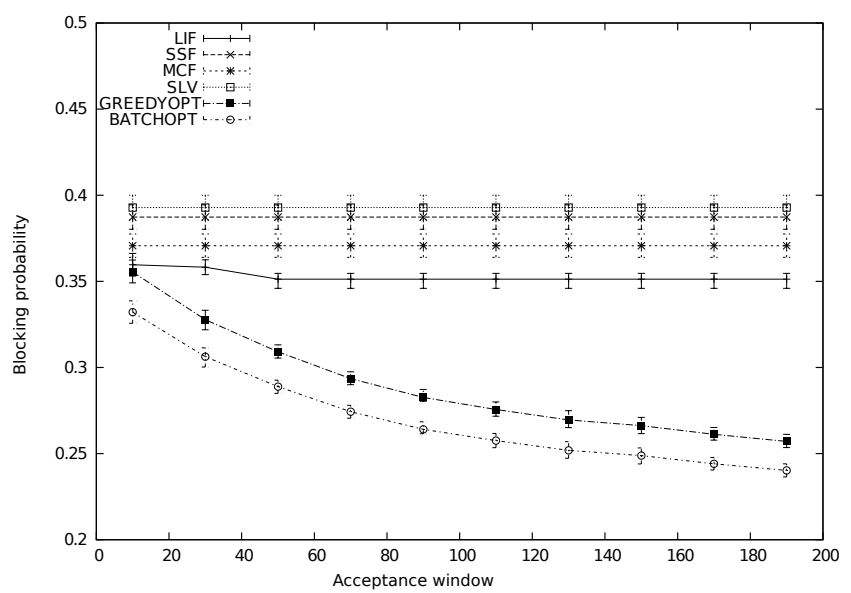

Figure 11: Blocking Probability as a function of the size of the acceptance window

Figure 11 shows the blocking probability as a function of the size of the acceptance window. In this experiment, as in [11], the difference between the highest and lowest offset time is adjusted to $50 \tau$ and the network load to $50 \tau$ with the acceptance window varying from $10 \tau$ to $190 \tau$.

It can be seen that, except for the BATCHOPT and GreedyOPT algorithms, the blocking probabilities produced do not depend on the increasing size of the acceptance window. In fact, the success of allocation by heuristics depends on the pattern of the starting and ending times of the requests rather than the number of requests.

Under BATCHOPT and GreedyOPT, the blocking probability decreases as the acceptance window increases, since increasing the acceptance window also increases the number of requests composing each batch. The optimal 
algorithms schedule the maximum number of requests in each batch resulting in lower blocking probabilities than those given by heuristics.

\subsection{Simulations using real network topologies}

In these experiments, the simulations used real topology networks such as NSFNet and Abilene, shown in Figure 12. Each link represents a fiber with 32 wavelengths with $2.5 \mathrm{Gbps}$ of capacity. The processing time for a control packet and the time required to configure the switching fabric was 50 $\mathrm{s}$. Each node at the edge can be either source or destination; for each request, source and destination were drawn from a uniform distribution. Traffic was generated according to a Poisson process, and the burst size followed a negative exponential distribution.

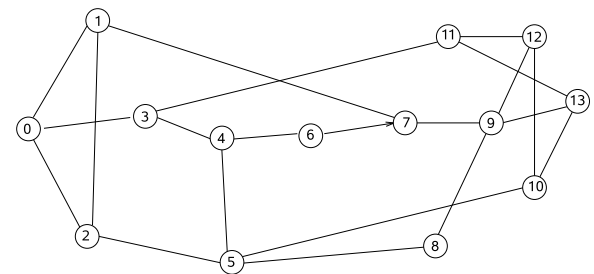

(a) Backbone NSFNet

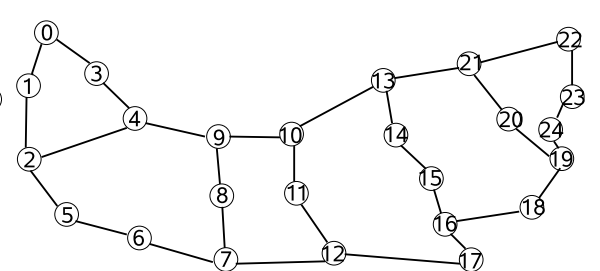

(b) Backbone Abilene

Figure 12: Real topologies used in the simulations

\subsubsection{Comparison of grouping strategies}

As previously discussed, the dimensioning of the acceptance window is challenging in complex topologies with several source-destination pairs. The scheduling algorithm used in these simulations was the BATCHOPT since it produced the best results in the evaluation reported in section 7.1. This algorithm was employed with JET- $\Delta$ strategy, as well as with the FIXED strategy proposed in [11].

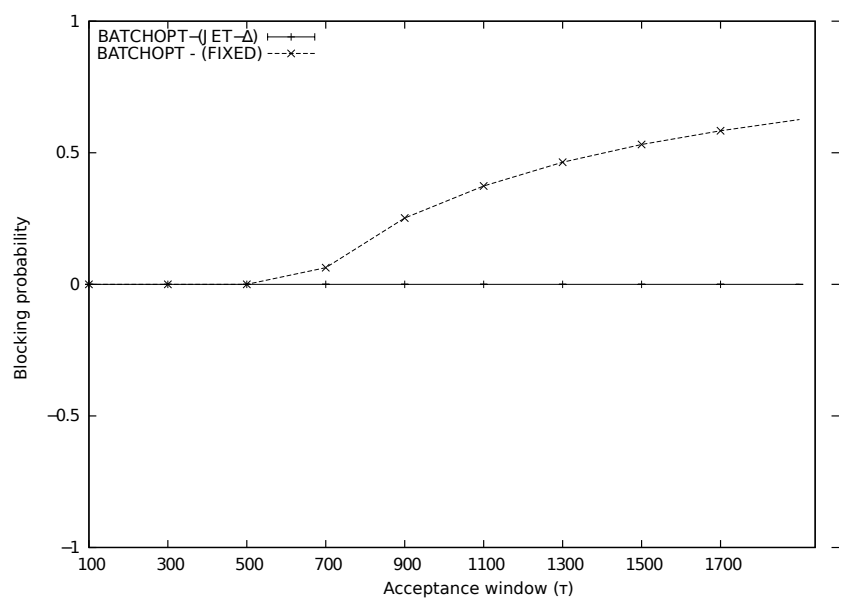

Figure 13: Blocking probability for the strategies of JET- $\Delta$ and FIXED as a function of acceptance window size.

Figure 13 displays the blocking probability as a function of the size of the acceptance window. While the acceptance window is smaller than the smallest offset time, the blocking probability produced by BATCHOPT is constant, regardless of the grouping strategy adopted. When the acceptance window becomes larger than the smallest offset time, the performance of FIXED deteriorates. Since the acceptance window is larger than the smallest possible offset time, the bursts corresponding to the requests in the batch arrive at the network nodes even before their requests have been processed, causing burst loss. 


\subsubsection{Comparison of the batch scheduling algorithms}

In this section, the two algorithms are evaluated using topologies of real network. In these experiments, JET- $\Delta$ was employed as grouping strategy.

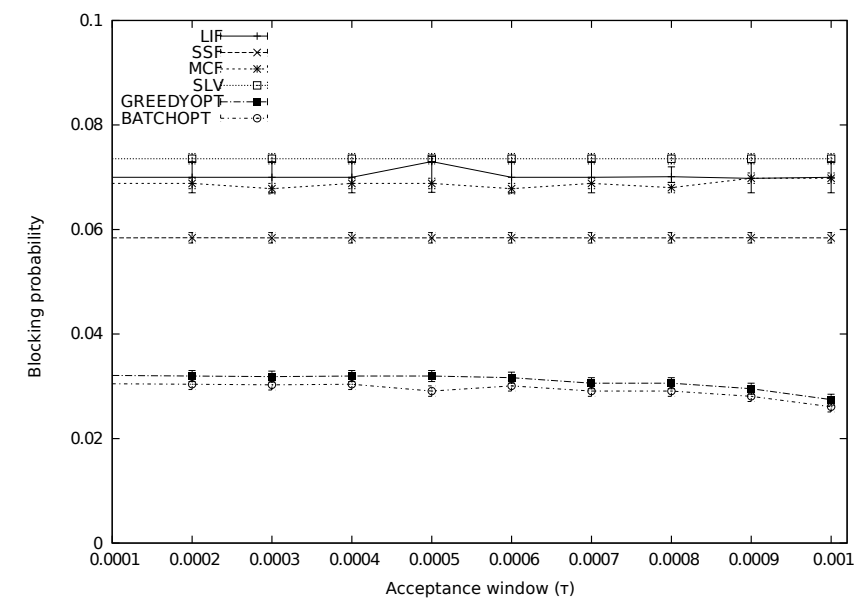

Figure 14: Blocking probability as a function of the window acceptance size.

First, the impact of the acceptance window size $(\Delta)$ was evaluated. Each request had a unit cost and the network load was 1000 Erlangs. Figure 14 shows the blocking probability as a function of $\Delta$ and Figure 15 shows the mean number of requests in each batch as a function of $\Delta$. The heuristics LIF, MCF, SSF and SLV were not sensitive to changes in the acceptance window size due to the fixed order in which the requests were processed, since the acceptance of requests depends more on the adjacency structure of the interval graph representing the requests than on the number of requests processed.

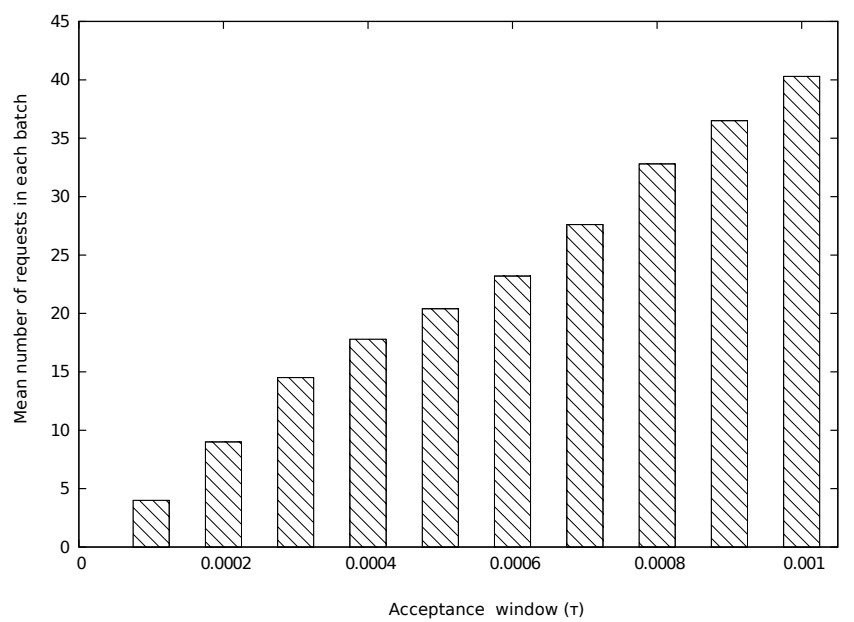

Figure 15: Average number of requests per batch as a function of acceptance window size.

Both, BATCHOPT and GreedyOPT can benefit from an increase in the mean number of requests in each batch. The larger the acceptance window for requests, the better is the performance. This is due to the fact that when more requests are processed at one time, more information about the intersections between requests is available. The algorithms can then make the best choice for scheduling the requests in the batch.

In practice, the window size should be adjusted as a function of the timing requirements of the traffic, given by Equation 6. In order to avoid impacting the performance of applications with time constraints, $\Delta$ was set to $1 m s$ [3]. 


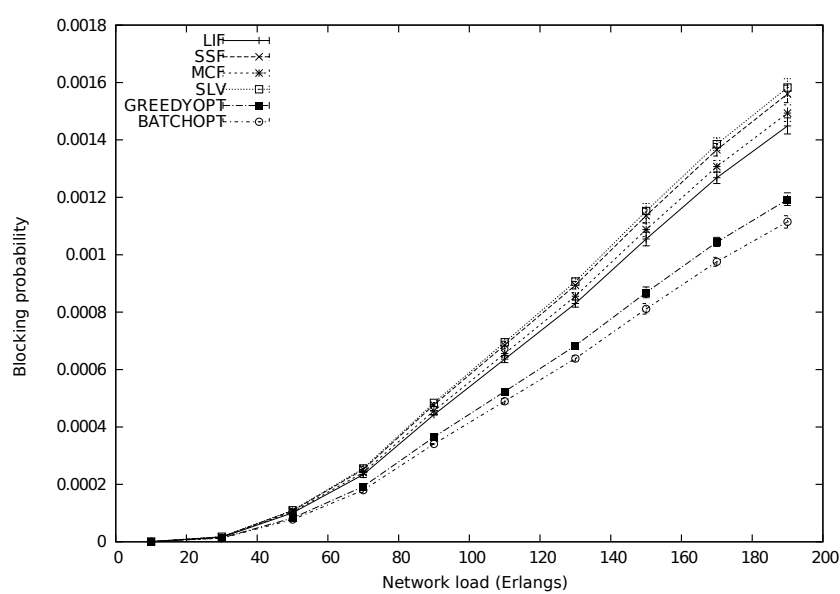

Figure 16: Blocking probability as a function of the network load.

Figure 16 reveals the blocking probability as a function of network load in a scenario where all requests have the same priority. Increasing the network load also increases the blocking probability. With the increase in network load, the size of maximal cliques of the interval graph associated with the requests increases, which explains the growth in the blocking probability. This is a common behavior in all algorithms evaluated. However, the increase in blocking probability is less when the BATCHOPT algorithm is used. Compared to the SLV algorithm (the algorithm that produced the worst results), the BATCHOPT algorithm produced gains of $42 \%$ in blocking probability. Since all requests had the same priority, the algorithm always select the set of requests of maximum cardinality and, thus, discards those with least number of request. The gains in the blocking probability of LIF, MCF and SSF in relation to SLV are $12 \%, 8 \%$ and $2 \%$, respectively.

The algorithm that produced the second lowest blocking probability was GreedyOPT, with a gain of $35 \%$ when compared to SLV. Although the GreedyOPT algorithm is optimum it does not guarantee that already scheduled requests remain scheduled i.e. a requests already scheduled can be blocked by those being processed in the current batch.

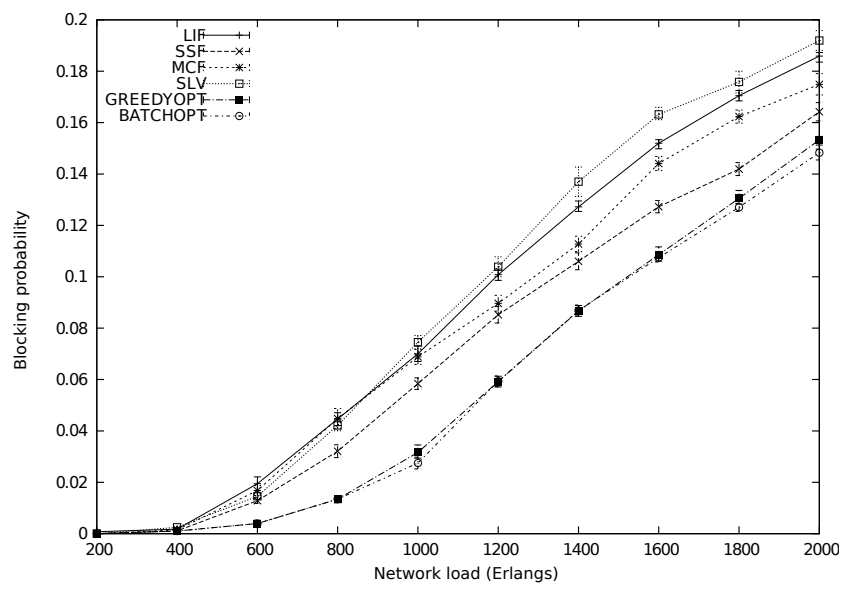

Figure 17: Blocking probability as a function of network load (considering QoS)

Simulations considering requests with different priorities (Quality of Service) were also conducted. In these simulations, five classes were considered with the class associated with a request being randomly picked from a uniform distribution. The weight of the classes involved follow the pattern: $w_{1}=1, w_{2}=2, w_{3}=4, w_{4}=8, w_{5}=16$, where $w_{i}$ is the weight of class $i$. 
Table 1: Relative gain in execution time

\begin{tabular}{|l|l|c|c|c|c|c|}
\hline & Batch 1 & Batch 2 & Batch 3 & Batch 4 & Batch 5 & Mean \\
\hline $\begin{array}{l}\text { Reference } \\
\text { value }\end{array}$ & $7.2 \times 10^{-2}$ & $8.0 \times 10^{-2}$ & $6.8 \times 10^{-2}$ & $7.6 \times 10^{-2}$ & $7.2 \times 10^{-2}$ & $7.3 \times 10^{-2}$ \\
\hline LIF & $37 \%$ & $27 \%$ & $22 \%$ & $40 \%$ & $33 \%$ & $32 \%$ \\
\hline SSF & $41 \%$ & $30 \%$ & $25 \%$ & $42 \%$ & $37 \%$ & $35 \%$ \\
\hline MCF & $33 \%$ & $26 \%$ & $26 \%$ & $34 \%$ & $25 \%$ & $28 \%$ \\
\hline SLV & $0.0 \%$ & $0.0 \%$ & $0.0 \%$ & $0.0 \%$ & $0.0 \%$ & $0.0 \%$ \\
\hline GreedyOPT & $36 \%$ & $27 \%$ & $25 \%$ & $40 \%$ & $31 \%$ & $32 \%$ \\
\hline BATCHOPT & $33 \%$ & $25 \%$ & $23 \%$ & $36 \%$ & $27 \%$ & $28 \%$ \\
\hline \hline
\end{tabular}

Figure 17 shows the blocking probability resulted from the algorithms when the requests have different priorities. Again, the algorithm that produced the lowest blocking probability was BATCHOPT algorithm followed by GreedyOPT algorithm. Moreover, the difference in the blocking probability among all evaluated algorithms is lower when compared to Figure 16. This happens because BATCHOPT and GreedyOPT try to maximize the sum of weights of the requests in the batch. Thus, while trying to do this, a higher number of requests with low weight can be discarded by those algorithms.

Figure 18 shows the distribution of loss per class. Note that BATCHOPT provides the lowest blocking probabilities for the classes with higher priorities, concentrating most of the losses in those with lowest priority. In other words, BATCHOPT was capable of providing differentiated services for distinct classes of service. This does not happen with the other policies, which leads to an "almost uniform" distribution of losses.

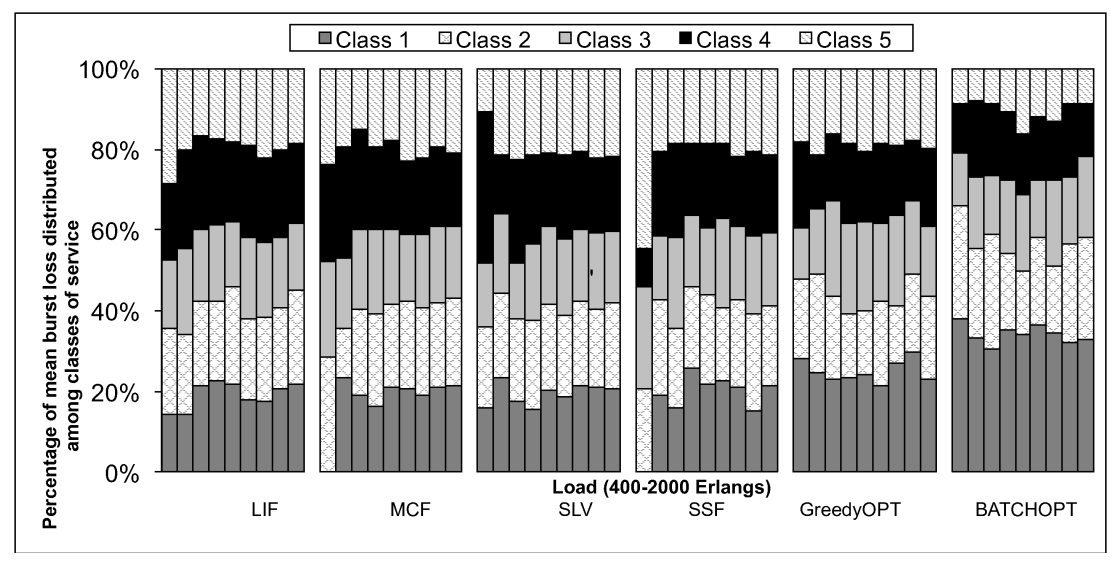

Figure 18: Blocking probability per class as a function of load.

The time for execution of the algorithms was also assessed. Simulations were run on a Intel Pentium Core 2 Duo machine with $2.8 \mathrm{Ghz}$ clock, 4GB RAM, and OpenSuse 11.1 operating system. The execution time was measured using the time command. Five sets of 500 requests were randomly generated. Ten simulations were executed for each set with the mean execution time for each set computed as well as the overall mean execution time. Gain was defined as the percentage difference in relation to the lowest execution time. They are displayed in Table 1 . The slowest algorithm is the SLV algorithm while the SSF is the fastest. GreedyOPT was at most 6\% slower than SSF. On an average it was 32\% faster than LIF algorithm and 3\% slower than the SSF. BATCHOPT was at most $10 \%$ slower than SSF. On an average it was $28 \%$ faster than LIF algorithm and $7 \%$ slower than SSF. 


\section{Conclusions}

This paper has introduced two optimum algorithms for the batch scheduling problem in OBS networks, they are based on a formulation of the problem of scheduling jobs with identical machines. Such a formulation considers not only the unprocessed requests but also those scheduled in the problem formulation. Although GreedyOPT has linear computational complexity, BATCHOPT produces the lowest blocking probability ratio. The blocking ratio given by these two algorithms are lower than those given by other existing heuristics. The difference between the proposed algorithms is that one (BATCHOPT) maintains the original scheduling of the already scheduled requests while the other (GreedyOPT) does not. Results derived via simulation show that this proposed BATCHOPT scheduling algorithm outperforms those previously proposed ones. BATCHOPT algorithm produces blocking probability $42 \%$ lower than that SLV, the algorithm with the highest blocking probability, and 30\% lower than LIF, the third lowest blocking probability. Moreover, the BACTHOPT algorithm is only 7\% slower than the SSF algorithm, which is the fastest heuristic. Moreover, BATCHOPT penalizes less the high priority classes in prioritized networks than do other existing heuristics. Furthermore, this paper has extended the JET reservation protocol for the consideration the scheduling of bursts although it remains compatible to the original JET.

\section{References}

[1] Qiao, C. and Yoo, M., Choices, Features and Issues in Optical Burst Swirching (OBS), Optical Network Magazine (1) (2000) pp. 36-44.

[2] Figueiredo, G. B., Control Mechanisms for Optical Burst Switched Networks, PhD Thesis, Institute of Computing, University of Campinas, 2009.

[3] Network QoS Needs of Advanced Internet Applications: A Survey, Internet2 QoS Working Group, Available in http://qos.internet2.edu/wg/apps/fellowship/Docs/Internet2AppsQoSNeeds.pdf, 2001.

[4] Sedgewick, Robert, Algorithms in C, Part 5: Graph Algorithms, Addison-Wesley Professional, 2001.

[5] Olariu, Stephan, An optimal greedy heuristic to color interval graphs, Information Procesing Letters 37 (1) 1991 pp. $21-25$.

[6] Bouzina, Khalid I. and Emmons, Hamilton, Interval Scheduling on Identical Machines, Journal of Global Optimization 9 (3-4) 1996 pp. 379-393.

[7] Rodrigues, Joel J. P. C. and Freire, Mario M and Lorenz Pascal, Impact of setup message processing and optical switch configuration times on the performance of IP over optical burst switching networks, Lecture notes in computer science 3733 (20) 2005 pp. $264-273$.

[8] Elhaddad, M. and Melhem, R. and Znati, T. and Basak, D., Traffic shaping and scheduling for OBS-based IP/WDM backbones, IEEE Opticom 52852003 pp. 336-345.

[9] Dovrolis, Constatinos and Stiliadis, Dimitrios, Proportional Differentiated Services: Delay differentiation and packet scheduling, IEEE Transactions on Networking, 10 (1) 2002 pp. 12-26.

[10] Rose, Donald. J. and Tarjan, Robert Endre and Leuker, George S., Algorithmic Aspects of vertex eliminations on graphs, SIAM Journal on Computing 5 (1976) pp. 266-283.

[11] Kaheel, A. and Alnuweiri, H., Batch Scheduling Algorithms for Optical Burst Switching Networks, Lecture notes in Computer Science 3462/2005 (2005) pp. 90-101.

[12] Charcranoon, S. and El-Bawab, T. S. and Cankaya, H. C. and Shin, J. D., Group Scheduling for Optical Burst Switched (OBS) Networks, IEEE Globecom (2003) pp. 2745-2749.

[13] Arkin, E. M. and Silverberg, E. B., Scheduling Jobs with Fixed Start and End Times, Discrete Applied Mathematics 18 (1987) pp. 1-8.

[14] Maranhao, J. and Soares, A. and Giozza, W. F., A study on architectures of OBS Networks, XXV Brazilian Symposium on Computer Networks 2007 pp. 133-146.

[15] Waldman, H. and Campelo, D. R. and Camelo, R., Dynamic Priority Strategies for Wavelength Assignment in WDM Rings, IEEE GLOBECOM 32000 pp. 1510-1514.

[16] Papoulis, A., Probability, Random Variables and Sthocastic Process, McGraw-Hill 2002.

[17] Moraes, I. M. and Cunha, D. O. and Bicudo, M. D. D. and Laufer, R. P. and Duarte, O. C. M. B., An Admission Control Mechanism for Providing Service Differentiation in Optical Burst-Switching Networks, Technical Report, Teleinformatics and Automation Group - Federal University of Rio de Janeiro, 2005,

[18] Zhang, Q. and Vokkarane, V. M. and Jue, J. P. and Chen, B., Absolute QoS Differentiation in Optical Burst-Switched Networks, IEEE Journal on Selected Areas in Communications 22 (2004) pp. 1781-1795.

[19] Liao, W. and Loi, C., Providing Service Differentiation for Optical-Burst Seitched Networks, Journal of Lightwave Technology 22 2004 pp. 1651-1660.

[20] Chen, Y. and Hamdi, M. and Tsang, D. H. K, Proportional QoS over OBS Networks, IEEE GLOBECOM 32001 pp. $1510-1514$.

[21] Liu, J. and Ansari, N. and Ott, T. J., FRR for Latency Reduction and QoS Provisioning in OBS Networks, Journal on Selected Areas in Communications 212003 pp. 1210-1219.

[22] Morato, D. and Aracil, J. and Diez, L. A., On Linear Prediction of Internet traffic for Packet and Burst Switchin Networks, IEEE ICC 2001 pp. 138-143.

[23] Li, J. and Qiao, C., Schedule Bursts Proactively for Optical Burst Switched Networks, Computer Networks 442004 pp. $617-629$.

[24] Wang, X. and Morikawa, H. and Aoyama, T., Priority-based wavelength Assignment Algorithm for Optical Burst Switched Photonic Networks, Optical Fiber Communications Conference 2002 pp. 765-766. 
[25] Chang, J. and Park, C., Efficient Channel Scheduling Algorithm in Optical Burst Switching Architecture, IEEE Workshop on High Performance Switching and Routing 2002 pp. 194-198.

[26] Vokkarane, V. M. and Jue, J. P., Prioritized Burst Segmentation and Composite Burst-Assembly Techniques for QoS Support in Optical Burst-Switched Networks, IEEE Journal on Selected Areas in Communications 212003 pp. 1198-1209.

[27] Detti, A. and Eramo, V. and Listanti, M., Performance Evaluation of a New Technique for IP Support in a WDM Optical Network: Optical Composite Burst Switching (OCBS), Journal of Lightwave Technology 202002 pp. 154-165.

[28] Xu, J. and Qiao, C. and Li, J. and Xu, G., Efficient Channel Scheduling Algorithms in Optical Burst Switched Networks, IEEE INFOCOM 3 2003 pp. 2268-2278.

[29] Xu, J. and Qiao, C. and li, J. and Xu, G., Efficient Burst Scheduling Algorithms in Optical Burst-Switched Networks Using Geometric Techniques, IEEE Journal on Selected Areas in Communications 222004 pp. 1796-1811.

[30] Li, J. and Qiao, C. and Chen, Y., Recent Progress in the Scheduling Algorithms in Optical-Burst-Switched Networks, OSA Journal of Optical Networking 32004 pp. 229-241.

[31] Chen, Y. and Qiao, C. and Xiang, Y., Optical Burst Switching (OBS): A New Area in Optical Networking Research, IEEE Network 18 2004 pp. 16-23.

[32] Oh, S. and Kang, M., A Burst Assembly ALgorithm in Optical Burst Switching Networks, Optical Fiber Communications Conference 2002 pp. 771-773.

[33] Dolzer, K., Assured Horizon - A New Combined Framework for Burst Assembly and Reservaton in Optical Burst Switched Networks, NOC 2002.

[34] Ge, An and Callegati, Franco and Tamil, Lakshman S., On Optical Burst Switching and Self-Similar Traffic, IEEE Communications Letters 42000 pp. $98-100$.

[35] Tachibana, Takuji and Ajima, Tamoya and Kasahara, Shoji, Round-Robin Burst Assembly and Constant Transmission Scheduling for Optical Burst Switching Networks, IEEE GLOBECOM 2003 pp. 2772-2776.

[36] Cao, Xiaojun and Li, Jikai and Chen, Yang and Qiao, Chumming, Assembling TCP/IP Packets in Optical Burst Switched Networks IEEE GLOBECOM 2002 pp. 2808-2812.

[37] Yu, Xiang and Chen, Yang and Qiao, Chumming, Study of Traffic Statistics of Assembled Burst Traffic in Optical Burst Switched Networks, Opticomm 2002 pp. 149-159.

[38] Yu, Xiang and Li, Jikai and Cao, Xiaojun and Chen, Yang and Qiao, Chumming, Traffic Statistics and Performance Evaluation in Optical Burst Switched Networks, Journal of Lightwave Technology 22 (12) 2004 pp. 2722-2738.

[39] Fumagalli, Andrea and Krishnamoorthy, Prasanna, A Low-Latency and Bandwidth-Efficient Distributed Optical Burst Switching Architecture for Metro Ring, ONDM 2003.

[40] Murty, C. and Gurusamy, M., WDM Optical Networks: Concepts, Design and Algorithms, Prentice Hall 2002

[41] Turner, J., Terabit Burst Switching, Journal of High Speed Networking 1999 pp. 3-16.

[42] Xiong, Y. and Vandenhoute, M. and Cankaya, C., Design and Analysis of Optical Burst-Switched Networks, SPIE'99 Conf. All Optical Networking: Architecture, Control and Management Issues 38431999 pp. 112-119.

[43] Xiong, Y. and Vandenhoute, M. and Cankaya, C., Control architecture in optical burst-switched WDM networks, IEEE Journal of Selected Areas on Communications 2000 pp. 1838-1851.

[44] Herzog, Martin and Maier, Martin and Reisslein, Martin, Metropolitan Area Packet-Switched WDM Networks: A Survey on Ring Systems, IEEE Communications Surveys 2004.

[45] Kaeel, Ayman and Alnuweiri, Hussein, Priority Scheme for Supporting Quality of Service in Optical Burst Switching Networks, Journal of Optical Networking 2004 pp. 707-719.

[46] Yoo, M. and Qiao, C., A New Optical Burst Switching Protocol for Supporting Quality of Service, All Optical Networking: Architecture, Control and Management Issue 1998 pp. 396-405.

[47] Xu, Lisong and Perros, Harry G. and Rouskas, George N., Access Protocols for Optical Burts-Switched Ring Networks, Information Sciences 1492003 pp. 75-81.

[48] Xu, Lisong and Perros, Harry G. and Rouskas, George N., A Simulation Study of Access Protocols for Optical Burst-Switched Ring Networks, Computer Networks 412003 pp. 143-160.

[49] Widjaja, I., Performance of burst admission control protocols, IEEE Proceding of Communications 1995 pp. 7-14.

[50] Haselton, E., A PCM Frame Switching Concept Leading to Burst Switching Network Architecture, IEEE Communications Magazine 21 1983 pp. 13-19.

[51] Perros, Harry G., An Introduction to ATM Networks, Wiley 2001.

[52] Cai, James and Fumagalli, Andrea, LightRIng: A distributed and contention-free bandwidth on-demand architecture, IFIP $5^{\text {th }}$ Working Conference on Optical Network Design ad Modeling 2001.

[53] Cai, James LightRing: An integrated WDM ring network solution for optical metropolitan area networks, PhD Thesis, Department of Electrical Engineering, The University of Texas at Dallas 2001.

[54] Qiao, C. and Yoo, M., Optical Burst Switching (OBS) - A new paradigm for an optical Internet, Journal of High-Speed Networks 8 (1) 1999 pp. 69-84.

[55] Qiao, C. and Yoo, M., Choices, Features and Issues in Optical Burst Swirching (OBS), Optical Network Magazine 12000 pp. $36-44$.

[56] D. West, "Introduction to Graph Theory" Prentice Hall, Inc., Upper Saddle River, NJ, (1996). 


\section{Appendix A.}

This appendix presents proofs related for the GreedyOPT algorithm.

Theorem 3. Let $k$ be the number of output channels of an OBS node, $n$ the number of incoming requests, $S$ the set of requests already allocated but with data bursts yet to arrive, and $s=|S|$, the cardinality of the set $S$. The computational complexity of the GreedyOPT algorithm is $O(N \log (N)+N k)=O(N \max (\log (N), k))$, where $N=n+s$.

Proof. It takes $O(s \log (s))$ to determine which unprocessed requests should be organized in a heap. It takes $O(N \log (N))$ to order the requests, as well as $O(N k)$ to verify the availability of a channel to accommodate the new request (line 4 of the GreedyOPT). Therefore, the complexity of GreedyOPT is $O(s \log (s)+N \log (N)+N k)$. Making $s=N$ in $s \log (s) \leq N \log (N)$, the complexity of GreedyOPT is thus $O(2 N \log (N)+N k)=O(N \log (N)+N k)=O(N(\log (N)+k))$. Considering the maximum value of the $(\log (N)+k)$, we have $O(N \max (\log (N), k))$ 


\section{Appendix B.}

This appendix provides proofs related to the BATCHOPT algorithm.

Theorem 5. The BATCHOPT algorithm optimally solves the OBS scheduling problem. Moreover, each already scheduled request remains scheduled on the scheduled channel.

Proof. Since each request in $S$ has a weight equal to infinity, and the algorithm computes a minimum cost flow, the algorithm will not use any of the arcs corresponding to requests in $S$. Therefore, the requests in $S$ will necessarily remain scheduled. Moreover, from the result of Theorem 4 it is known that among the requests in $I$, the algorithm will schedule a subset $I^{\prime}$ of requests of maximum weight.

Indeed, it is not necessary to change the channels for it is assigned to requests that have already been scheduled. Suppose the algorithm generates the schedule starting at some time $t$. Let $S^{\prime} \subseteq S$ be the set of requests with starting time prior to $t$. This means that each request in $S^{\prime}$ is already scheduled, and the scheduled channel can not be changed. But notice that for the requests in $S \backslash S^{\prime}$ it is indeed possible to change their channel without problems. The BATCHOPT algorithm selects a set $I^{\prime}$ of new requests such that for any time $t^{\prime} \geq t$ there are at most $k$ requests from $I^{\prime} \cup S$ that will be transmitted at time $t^{\prime}$. The requests in $I^{\prime} \cup S$ can be sorted by their starting times and scheduled in this order on an available channel. This generates a feasible schedule since at any time $t^{\prime}$ there are at most $k$ requests intersecting it, and will thus be there an available channel each time a request is assigned. Notice that the requests in $S$ were previously scheduled in a feasible schedule. Since the requests in $S^{\prime}$ have the earliest starting times among the requests in $S \cup I^{\prime}$, they are processed first so that each request in $S^{\prime}$ can be scheduled on the previously scheduled channel.

Theorem 6. The BATCHOPT algorithm has time complexity $O\left(N^{2} \log (N)+N\right)$, where $N=|I \cup S|$, i.e, $N$ is the number of requests in the batch plus the number of requests in $S$.

Proof. Let $n$ be the size of the incoming batch $(n=|I|)$ and let and $s$ be the size of previously scheduled requests that intersects with $I(s=|S|)$.

The time complexity to find the set $S$ is $O(s \log s)$ if previously scheduled requests are stored using a heap. The AS algorithm has time complexity $O\left(N^{2} \log N\right)$ (see [13]), and since $N=s+n$ the overall complexity to find $S$ and to schedule $I \cup S$ is $O\left(N^{2} \log N\right)$. 